SubmitTed TO ApJ

Preprint typeset using $\mathrm{LAT}_{\mathrm{E}} \mathrm{X}$ style emulateapj v. 05/12/14

\title{
STELLAR LOCI III: PHOTOMETRIC METALLICITIES FOR HALF MILLION FGK STARS OF STRIPE 82
}

\author{
Haibo Yuan $^{1,2}$, XiaOwei Liu ${ }^{3,1}$, MaOsheng Xiang ${ }^{3}$, Yang Huang ${ }^{3}$, BingQiu Chen ${ }^{3,2}$ \\ Received ; accepted
}

\begin{abstract}
We develop a method to estimate photometric metallicities by simultaneously fitting the dereddened colors $u-g, g-r, r-i$ and $i-z$ from the SDSS with those predicted by the metallicity-dependent stellar loci. The method is tested with a spectroscopic sample of main-sequence stars in Stripe 82 selected from the SDSS DR9 and three open clusters. With 1 per cent photometry, the method is capable of delivering photometric metallicities precise to about $0.05,0.12$, and 0.18 dex at metallicities of 0.0 , -1.0 , and -2.0 , respectively, comparable to the precision achievable with low-resolution spectroscopy at a signal-to-noise ratio of 10. We apply this method to the re-calibrated Stripe 82 catalog and derive metallicities for about 0.5 million stars of colors $0.3<g-i<1.6 \mathrm{mag}$ and distances between $0.3-18$ kpc. Potential systematics in the metallicities thus derived, due to the contamination of giants and binaries, are investigated. Photometric distances are also calculated. About 91, 72, and 53 per cent of the sample stars are brighter than $r=20.5,19.5$, and $18.5 \mathrm{mag}$, respectively. The median metallicity errors are around $0.19,0.16,0.11$, and 0.085 dex for the whole sample, and for stars brighter than $r=$ 20.5, 19.5, and 18.5 mag, respectively. The median distance errors are 8.8, 8.4, 7.7, and 7.3 per cent for the aforementioned four groups of stars, respectively. The data are publicly available. Potential applications of the data in studies of the distribution, (sub)structure, and chemistry of the Galactic stellar populations, are briefly discussed. The results will be presented in future papers.
\end{abstract}

Keywords: methods: data analysis - stars: fundamental parameters - stars: general - surveys

\section{INTRODUCTION}

Metallicity is one of the most important stellar parameters. Together with the initial mass and age, they fully determine the current properties of a (single) star, including the internal structure and atmospheric spectrum. The atmospheric metallicities of long-lived latetype stars retain a fossil record of the chemical composition of the interstellar medium at the time and place of their formation. Measuring the metallicity distribution of stars in the Milky Way provides important clues to the formation, and chemical and dynamical evolution of the Galaxy (e.g., Casagrande et al. 2011; Schlesinger et al. 2012; An et al. 2013; Peng et al. 2013).

Metallicities, along with other stellar parameters, have now been measured for millions of stars from low- to medium- resolution spectra collected by multi-fiber spectroscopic surveys, including the Radial Velocity Experiment (RAVE; Steinmetz et al. 2006), the Sloan Digital Sky Survey (SDSS; York et al. 2000) and the LAMOST Galactic surveys (Deng et al. 2012; Zhao et al. 2012; Liu et al. 2014). With state-of-the-art stellar parameter pipelines such as the SEGUE Stellar Parameter Pipeline (SSPP; Lee et al. 2008a,b) developed for the Sloan Extension for Galactic Understanding and Exploration (SEGUE; Yanny et al. 2009), the LAMOST Stellar Parameter Pipeline (LASP; Wu et al. 2014, in preparation), and the LAMOST Stellar Parameter Pipeline at Peking University (LSP3; Xiang et al. 2015), spectroscopic metallicities can be determined to an accuracy

\footnotetext{
${ }^{1}$ Kavli Institute for Astronomy and Astrophysics, Peking University, Beijing 100871, P. R. China; email: yuanhb4861@pku.edu.cn, x.liu@pku.edu.cn

${ }^{2}$ LAMOST Fellow

${ }^{3}$ Department of Astronomy, Peking University, Beijing 100871, P. R. China
}

of about 0.1 dex at good $(\gtrsim 10)$ signal-to-noise ratios (SNRs). In spite of the high accuracy, of the hundreds of billions of Galactic stars spreading over the whole sky of $4 \pi$ steradians, the number that can be spectroscopically targeted within the limiting magnitudes of the surveys, albeit now rapidly increased to order of a few millions thanks to the operation of LAMOST (Cui et al. 2012), remains to be small. For example, the SDSS has hitherto spectroscopically surveyed hundreds of thousands of stars selected with a variety of algorithms in several hundred pencil beams. With 4,000 fibers, LAMOST has the potential to build a statically complete sample of millions of stars selected with a simple yet non-trivial algorithm (Yuan et al. 2015a), allowing the recovery of the underlying population for whatever class of objects that are revealed by the spectra (e.g., Rebassa-Mansergas et al. 2014). Even so, various selection effects have still to be properly taken into account, which is a non-trivial task.

Stellar colors depend mainly on star's effective temperature. However, they also vary significantly with metallicity, in particular those blue colors as most metal absorption lines are in the blue wavelength range. Therefore, photometric colors have long been used to estimate stellar metallicities, for example, in the traditional UV excess method (Wallerstein 1962; Sandage \& Smith 1963). For FG main-sequence (MS) stars of the same $B-V$ color, those of bluer $U-B$ colors have lower metallicities. The method has been extended to the SDSS photometric system by Ivezić et al. (2008) using the SDSS DR6 data. In spite of successfully mapping the metallicity distribution for a complete volume-limited sample of 2 million FG stars at distances between 500 pc and $8 \mathrm{kpc}$, the photometric metallicity estimators developed by Ivezić et al. have some limitation and room of refinement: a) The estimators are only applicable to FG stars 
of $g-r$ colors between $0.2-0.6$ mag; b) The calibration of $[\mathrm{Fe} / \mathrm{H}]$ estimates yielded by the SSPP has improved significantly since DR7, particularly for those very metalrich and very metal-poor stars (Lee et al. 2008a, 2008b; Allende Prieto et al. 2008; Smolinski et al. 2011); c) The estimators are built on colors $u-g$ and $g-r$ only. Employing as many colors as possible can potentially tighten the constraints and reduce the errors of metallicity estimates. For example, An et al. (2013) use the SDSS ugriz photometry and empirically calibrated stellar isochrones to drive temperatures, metallicities, and distances of individual stars.

The repeatedly scanned equatorial Stripe $82(|\mathrm{Dec}|<$ $\left.1.266^{\circ}, 20 \mathrm{~h} 34 \mathrm{~m}<\mathrm{RA}<4 \mathrm{~h} 00 \mathrm{~m}\right)$ has delivered accurate photometry for about one million stars in $u, g, r, i, z$ bands (Ivezic et al. 2007). This is the largest single uniform data set publicly available with optical photometry internally consistent at 1 per cent level, providing "a practical definition of the SDSS photometric system" (Ivezić et al. 2007). Using the spectroscopic information of about 24,000 stars in the Stripe available from SDSS Data Release 9 (DR9; Ahn et al. 2012), Yuan et al. (2015b) have further re-calibrated the Stripe 82 photometric catalog with an innovative spectroscopy based stellar color regression (SCR) method, achieving an unprecedented internal calibration accuracy of about 0.005 , $0.003,0.002$, and $0.002 \mathrm{mag}$ in colors $u-g, g-r, r-i$, and $i-z$, respectively. By combining the spectroscopic information and the re-calibrated photometry of Stripe 82, Yuan et al. (2015c, hereafter Paper I) have constructed a large, clean sample of MS stars with well determined metallicities and extremely accurate colors to derive metallicity-dependent stellar loci in the SDSS colors and investigate their intrinsic widths.

Compared to previously derived stellar color loci (e.g., Covey et al. 2007; Davenport et al. 2014; Chen et al. 2014), metallicity-dependent stellar loci, as discussed in Paper I, have a number of potential applications that have already led to some interesting results. For example, in Paper II (Yuan et al. 2015d), we propose a Stellar Locus OuTlier (SLOT) method and provide a modelindependent estimate of the binary fraction for field FGK stars. In this third paper of the series, we develop a method to obtain accurate photometric metallicities for stars of a wide range of color, and apply the method to the re-calibrated Stripe 82 catalog.

The paper is organized as follows. In Section 2, we introduce the photometric data and method. Various tests of the method are presented in Section 3. The results are presented in Section 4. The summary is given in Section 5, along with a brief discussion of some potential applications of the newly deduced metallicities.

\section{DATA AND METHOD}

\subsection{Data}

The re-calibrated Stripe 82 catalog contains 1,006,849 targets. All the targets have $g, r$, and $i$ band magnitudes, but only 593,510 targets of them have $u$ band magnitudes, given that many red stars are too faint to be detected in $u$ band. Also there are 157 targets without $z$ band magnitudes. The stars are firstly dereddened using the dust reddening map of Schlegel et al. (1998; SFD98 hereafter) and the empirical reddening co- efficients of Yuan et al. (2015b), derived using a star pair technique (Yuan, Liu \& Xiang 2013). Given that color $u-g$ is most sensitive to metallicity, only stars that have a dereddened color $0.55 \leq g-i \leq 1.2 \mathrm{mag}$ and all $u, g, r, i$, and $z$ magnitudes are included in the current analysis. This yields a sample of 469,112 stars in total.

Panels (a) - (e) of Fig 1 plot the photometric errors as a function of the observed (undereddened) magnitudes for the sample stars. For $u$ band, the photon counting noises start to dominate the errors at $u \gtrsim 19.0$ mag. The errors are about $0.01 \mathrm{mag}$ at $u=19.0 \mathrm{mag}$ and 0.1 mag at $u=22.0 \mathrm{mag}$. For $g, r$, and $i$ bands, the errors are dominated by the calibration uncertainties and essentially constant, at the level of 0.006, 0.005, and 0.005 mag for stars brighter than about $g=20.0, r=19.0$, and $i=19.0 \mathrm{mag}$, respectively. At the detection limits ( $g \sim 22.5 \mathrm{mag}, r \sim 21.5 \mathrm{mag}$, and $i \sim 21.0 \mathrm{mag}$ ), the errors increase to about $0.05 \mathrm{mag}$. For $z$ band, the photon counting noises dominate at $z \gtrsim 18.0 \mathrm{mag}$, and the errors are about $0.01,0.02,0.05$, and $0.1 \mathrm{mag}$ at $z=$ 18, 19, 20, and $21 \mathrm{mag}$, respectively. Panels (f) and (g) show the sample distributions in the $(g-i)-(u-g)$ and $(g-i)-(g-r)$ planes, respectively. The stellar loci of metallicities $[\mathrm{Fe} / \mathrm{H}]=-2.0$ and 0.0 as derived in Paper I are also over-plotted. Most stars in the sample have an $[\mathrm{Fe} / \mathrm{H}]$ value between -2.0 and 0.0 . Panels (h) and (i) show the sample distributions in the $(g-i)-r$ and $(u-g)-r$ color-magnitude diagrams. Clearly, the sample is a $u$-band limited one and contains more blue stars than red ones.

\subsection{Method}

We use a minimum $\chi^{2}$ technique to determine metallicity. Here $\chi^{2}$ is defined as:

$$
\begin{array}{r}
\chi^{2}([\mathrm{Fe} / \mathrm{H}], g-i)= \\
\sum_{i=1}^{4} \frac{\left[c_{\mathrm{obs}}^{i}-R_{c}^{i} \times E(B-V)-c_{\mathrm{int}}^{i}([\mathrm{Fe} / \mathrm{H}], g-i)\right]^{2}}{\left(\sigma_{c}^{i}\right)^{2} \times(4-2)}
\end{array}
$$

where $c_{\text {obs }}^{i}(i=1-4)$ are the observed colors and $i=1,2$, 3,4 for colors $u-g, g-r, r-i$ and $i-z$, respectively. $R_{c}^{i}$, $c_{\text {int }}^{i}([\mathrm{Fe} / \mathrm{H}], g-i)$, and $\sigma_{c}^{i}(\mathrm{i}=1-4)$ are respectively the reddening coefficients from Yuan et al. (2015b), the intrinsic colors predicted by the metallicity-dependent stellar loci for a given set of metallicity $[\mathrm{Fe} / \mathrm{H}]$ and intrinsic color $g-i$, and the uncertainties of observed colors. Values of $E(B-V)$ are from the SFD98 extinction map. $R_{c}^{i}=1.060,1.055,0.604$, and 0.528 , for $i=1,2,3$, and 4 , respectively. Values of $\sigma_{i}$ are estimated from the magnitude errors and calibration uncertainties. The calibration uncertainties are adopted as 0.005, 0.003, 0.002 and $0.002 \mathrm{mag}$ in colors $u-g, g-r, r-i$ and $i-z$, respectively (Yuan et al. 2015b).

We use a brute-force algorithm to determine the optimal $[\mathrm{Fe} / \mathrm{H}]$ and intrinsic $g-i$ color for each sample star. For a given sample star, the value of $[\mathrm{Fe} / \mathrm{H}]$ is varied from -2.5 to 0.5 at a step of $0.01 \mathrm{dex}$, and the value of $g-i$ is varied from $(g-i)_{\mathrm{obs}}-2 \times \sigma_{g-i}$ to $(g-i)_{\mathrm{obs}}+2 \times \sigma_{g-i}$ (mag) at a step of $0.2 \times \sigma_{g-i}$ mag. A $301 \times 21$ array of

4 All colors and magnitudes quoted refer to the dereddened values unless specified otherwise. 

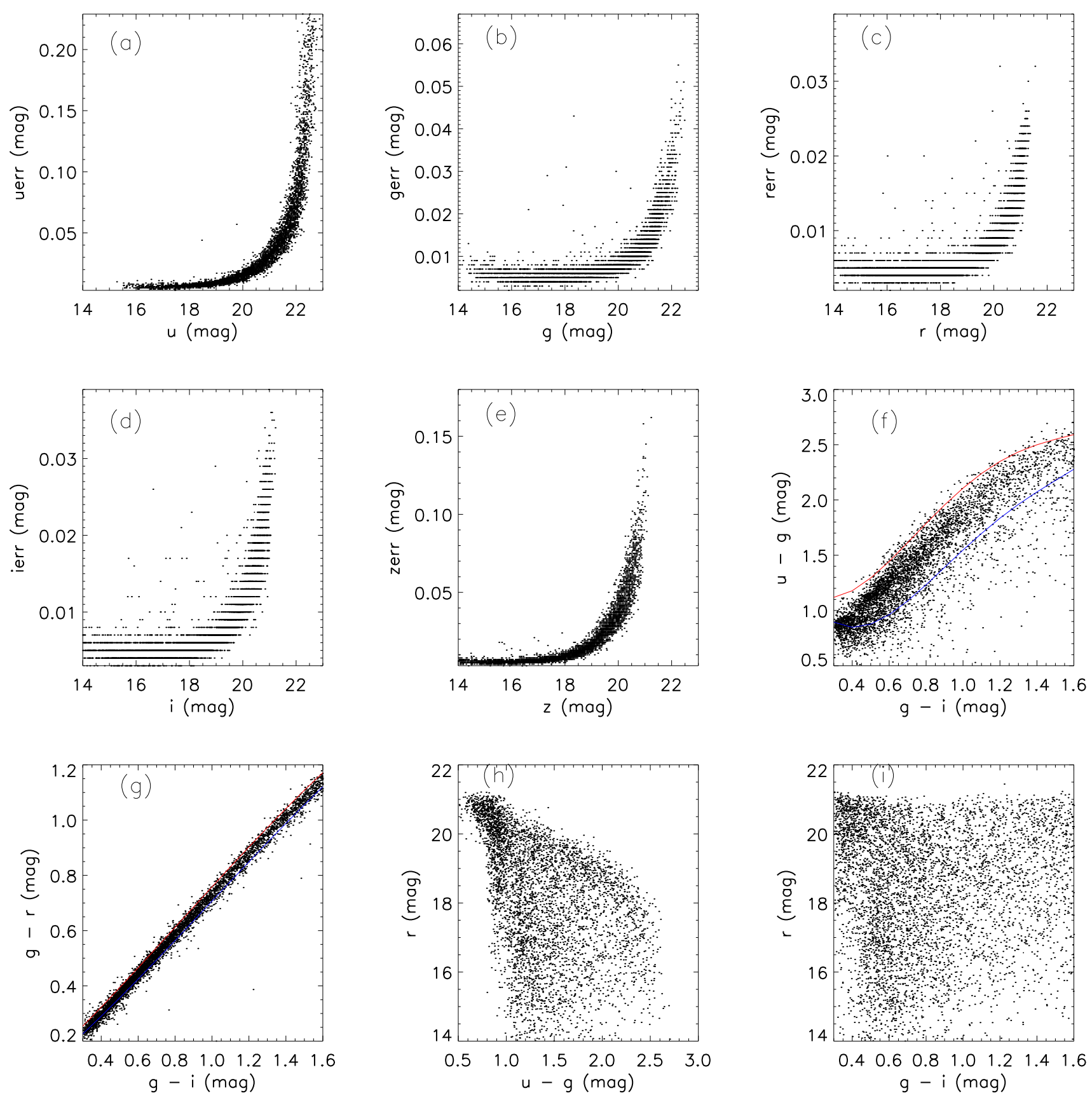

Figure 1. Distributions of photometric errors as a function of observed magnitudes [Panels (a) - (e)] and distributions of sample stars in the $(g-i)-(u-g)(\mathrm{f}),(g-i)-(g-r)(\mathrm{g}),(g-i)-r(\mathrm{~h})$, and $(u-g)-r$ (i) planes. In Panels (f) and (g), the blue and red lines denote the stellar loci for $[\mathrm{Fe} / \mathrm{H}]=-2.0$ and 0.0 , respectively. To avoid crowdness, only one in a hundred randomly selected targets are plotted.

$\chi^{2}$ values is calculated to find the minimum value of $\chi^{2}$, $\chi_{\min }^{2}$, and the corresponding values of intrinsic $g-i$ color, $(g-i)_{0}$, and of metallicity $[\mathrm{Fe} / \mathrm{H}]$. The error of $[\mathrm{Fe} / \mathrm{H}]$ is estimated as $1 \sigma$ uncertainty that corresponds to the difference of $[\mathrm{Fe} / \mathrm{H}]$ values at $\chi_{\min }^{2}$ and $\chi_{\min }^{2}+1$ (Avni 1976; Wall 1996).

\section{TESTS OF THE METHOD}

\subsection{Internal test}

We first apply the technique to the spectroscopic sample of Stripe 82 used to construct the metallicitydependent stellar loci in Paper I, and compare the resul- tant photometric metallicities with the spectroscopic values. The spectroscopic sample of Paper I contains 24,492 stars of a spectral SNR $>10$, a line-of-sight extinction $E(B-V) \leq 0.15 \mathrm{mag}$, a surface gravity $\log g \geq 3.5$ dex, a color $0.3 \leq g-i \leq 1.6 \mathrm{mag}$, an effective temperature $T_{\text {eff }} \geq 4,300 \mathrm{~K}$, and a metallicity $-2.0 \leq[\mathrm{Fe} / \mathrm{H}] \leq 0.0$. In the current work, we have relaxed the cut in $[\mathrm{Fe} / \mathrm{H}]$ and included another 1,173 stars with a $[\mathrm{Fe} / \mathrm{H}]$ value either larger than 0.0 or lower than -2.0 to allow us test the validity range of $[\mathrm{Fe} / \mathrm{H}]$ of the method. The final test sample thus contains 25,665 stars.

The top left panel of Fig. 2 shows a histogram distri- 
bution of $\chi_{\min }^{2}$ values of the sample. Only 11.4, 4.8, and 1.3 per cent of the stars show a $\chi_{\min }^{2}$ value larger than 3 , 5 , and 10, respectively. Most stars showing a large $\chi_{\min }^{2}$ value are probably binaries, as binaries generally do not follow the stellar loci of single (MS) stars (Yuan et al. 2015d). The top right panel of Fig. 2 displays a comparison of the photometric metallicities thus derived and the corresponding spectroscopic values taken from the DR9. There is no significant systematic difference, and the dispersion is only 0.15 dex. The random errors of $[\mathrm{Fe} / \mathrm{H}]$ in the DR9 as yielded by the SSPP pipeline have been determined as a function of spectral SNR and $[\mathrm{Fe} / \mathrm{H}]$ for FGK MS stars using duplicate observations (Yuan et al. $2015 \mathrm{~d})$. The random errors of $[\mathrm{Fe} / \mathrm{H}]$ for the test sample stars range from 0.03 to 0.20 dex, with a median value of 0.08 dex. The result suggests that the photometric metallicities derived in the current work have a typical error of 0.13 dex. Note that for $\mathrm{SNR}=10$, the random errors of $[\mathrm{Fe} / \mathrm{H}]$ yielded by the SSPP are about $0.11,0.16$, and 0.19 dex at $[\mathrm{Fe} / \mathrm{H}]=0,-1$, and -2 , respectively. The bottom two panels of Fig. 2 display the differences between the photometric and spectroscopic metallicities as a function of metallicity $[\mathrm{Fe} / \mathrm{H}]$ and color $g-i$. The sample is divided into bins of $[\mathrm{Fe} / \mathrm{H}]$ and $g-i$. The median and dispersion values for individual bins are overplotted. No systematic dependences of the differences on $[\mathrm{Fe} / \mathrm{H}]$ are found within the $[\mathrm{Fe} / \mathrm{H}]$ range of $-2.0-$ 0.0 , and on color $g-i$ within the range of the plot. Not surprisingly, at $[\mathrm{Fe} / \mathrm{H}]>0.0$, the photometric metallicities are under-estimated by about 0.15 dex. At $[\mathrm{Fe} / \mathrm{H}]<$ -2.0 , the photometric metallicities are over-estimated by about 0.2 dex. Note the boundary in the top left corner of the bottom left panel corresponds to the lower limit of photometric metallicities treated in the current work, i.e., -2.5 . For stars of $g-i \lesssim 0.35 \mathrm{mag}$ and $[\mathrm{Fe} / \mathrm{H}] \lesssim$ -1.5 , the metallicity-dependent stellar loci of different metallicities intersect, leading to approximately constant $[\mathrm{Fe} / \mathrm{H}]$ value around -2.2 . This artifact can be clearly seen in the bottom left panel of Fig. 2. This is also the reason why the bottom right panel shows large scatters at the bluest colors.

The uncertainties of photometric metallicity depend on the apparent magnitude, metallicity and color of the star under consideration. For the test sample, the median uncertainty is 0.10 dex and the mean value is 0.13 dex. This is consistent with the typical error of 0.13 dex as yielded by comparing the photometric and spectroscopic metallicities. Fig. 3 plots the estimated uncertainties of photometric $[\mathrm{Fe} / \mathrm{H}]$ of the test sample against the uncertainties of color $u-g$, against the spectroscopic $[\mathrm{Fe} / \mathrm{H}]$ from the DR9, and color $g-i$, respectively. A good, linear correlation is seen between the uncertainties of photometric $[\mathrm{Fe} / \mathrm{H}]$ and the errors of $u-g$, as can be expressed as,

$$
\sigma([\mathrm{Fe} / \mathrm{H}])=4.25 \times \sigma(u-g)+0.034 .
$$

A 0.02 mag error in $u-g$ color yields a 0.12 dex uncertainty in $[\mathrm{Fe} / \mathrm{H}]$, in line with the variation of $u-g$ color to the metallicity (Paper I). The $[\mathrm{Fe} / \mathrm{H}]$ uncertainty is anti-correlated with $[\mathrm{Fe} / \mathrm{H}]$,

$$
\sigma([\mathrm{Fe} / \mathrm{H}])=-0.074 \times[\mathrm{Fe} / \mathrm{H}]+0.043 .
$$

Such an anti-correlation is expected. For a given uncertainty of $u-g$, the stellar loci are more sensitive to
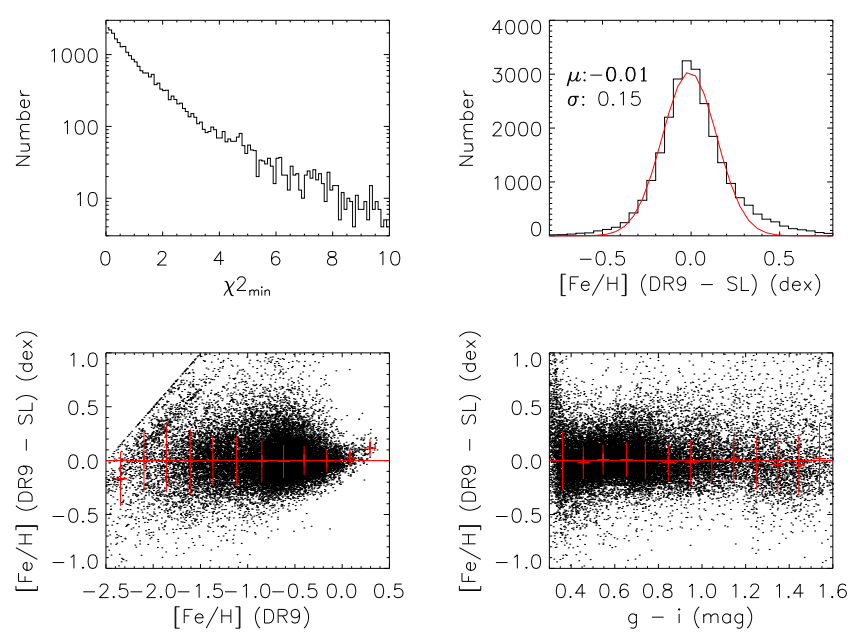

Figure 2. Top panels: Histogram distributions of $\chi_{\min }^{2}$ values of the test sample (left) and the differences of photometric metallicities derived in the current work and the corresponding spectroscopic values from the DR9 (right). The red curve denotes a Gaussian fit, with the mean and dispersion values labeled. Bottom panels: Differences between the photometric and spectroscopic metallicities are plotted as a function of spectroscopic metallicity (left) and of $g-i$ color (right). The sample is divided into bins of $[\mathrm{Fe} / \mathrm{H}]$ and $g-i$ of widths $0.25 \mathrm{dex}$ and $0.1 \mathrm{mag}$, respectively. For each bin, the median and dispersion values are over-plotted in red.
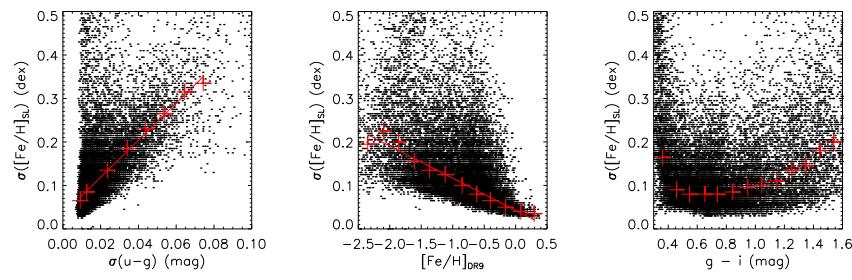

Figure 3. Uncertainties of photometric $[\mathrm{Fe} / \mathrm{H}]$ of the test sample plotted against errors of $u-g$ color (left), spectroscopic values of $[\mathrm{Fe} / \mathrm{H}]$ from DR9 (middle), and color $g-i$ (right). The sample is divided into bins of $\sigma(u-g),[\mathrm{Fe} / \mathrm{H}]$, and $g-i$ of widths $0.01 \mathrm{mag}$, $0.25 \mathrm{dex}$, and $0.1 \mathrm{mag}$, respectively. The median and dispersion values of the photometric estimates of $[\mathrm{Fe} / \mathrm{H}]$ of the individual bins are over-plotted in red. The red lines in the left and middle panels denote linear fits to those median values.

metallicity at higher metallicities (Paper I). Note that the constants in Eqs. (2) and (3) are valid only for the test sample, for which the median error in $u-g$ color is about $0.013 \mathrm{mag}$ and the median $[\mathrm{Fe} / \mathrm{H}]$ is -0.71 . The right panel of Fig. 3 illustrates that photometric estimation of metallicity works best for stars of $g-i$ colors between 0.4 $-1.2 \mathrm{mag}$. Note also that for individual stars, the estimated errors in some cases could be significantly underestimated.

For stars of the test sample of $g-r$ color between 0.2 - $0.6 \mathrm{mag}$, we have also determined their photometric metallicities using Eq. (4) of Ivezić et al. (2008). Fig. 4 compares the results from the current work, those deduced using the relation of Ivezić et al., and the spectroscopic values from the DR9 for this sub-sample of stars. Compared to the relation of Ivezić et al., out current method not only yields much smaller discrepancies $(0.14$ versus 0.19 dex on average) when comparing with the spectroscopic values, but also is applicable to a much wider color range. The strength of the current method 

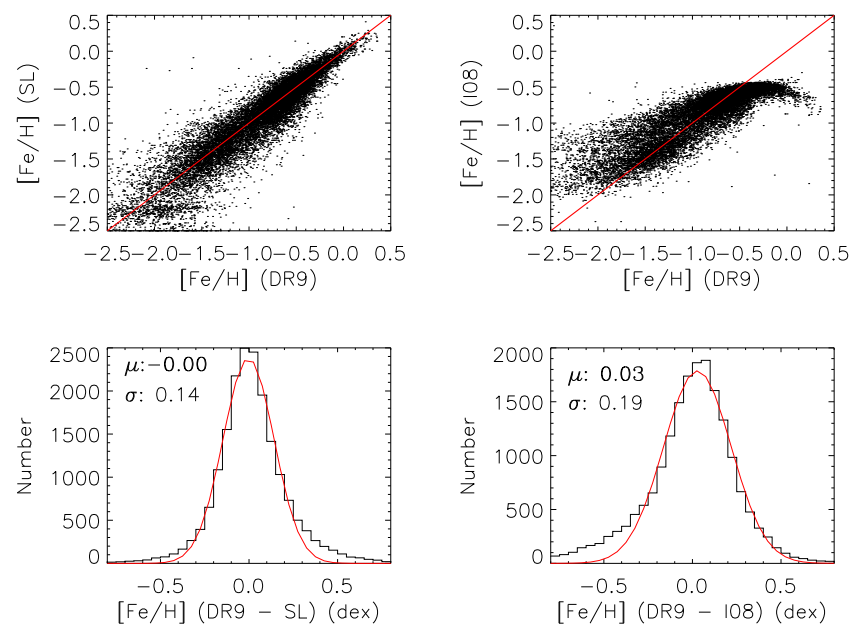

Figure 4. Comparisons of photometric metallicities derived in the current work (left panels) and those using the relation of Ivezić et al. (2008) (right panels) with the spectroscopic values from the DR9 for a sub-sample of test stars of $0.2<g-r<0.6$ mag. The diagonal lines in the top panels denote equality of the two quantities in comparison. The red curves in the bottom panels denote Gaussian fits to the distributions of the differences, with the mean and dispersion values labeled.

comes from the fact that it makes use of as much information as available. The systematics of results yielded by the relation of Ivezić et al. at metallicities higher than -0.5 or lower than -1.5 is possibly caused by the potential metallicity calibration problems in the SDSS DR6.

\subsection{Test with star clusters}

We have applied the method to three open clusters (OCs; NGC 2420, M 67, and NGC 6791), using the SDSS photometry from An et al. (2008). The $[\mathrm{Fe} / \mathrm{H}]$ estimates of the OCs NGC 2420, M 67, and NGC 6791 have been previously determined by spectroscopy at -0.37 (Anthony-Twarog et al. 2006), 0.0 (An et al. 2007), and 0.47 (Carretta et al. 2007), respectively. The candidate member stars are selected based on the spatial positions as well as the distributions in the $g-i$ versus $r$ color-magnitude diagram (Xiang et al. 2015). The stars are then dereddened using the SFD extinction map and the empirical reddening coefficients of Yuan, Liu \& Xiang (2013). Only MS stars are selected.

The results are showed in Fig 5 . For NGC 2420, the mean photometric metallicity is $-0.29 \pm 0.13,0.02 \mathrm{dex}$ higher than the mean spectroscopic value from the DR9 and 0.08 dex larger than the literature value. The photometric metallicities show some trend with $g-i$ color in the sense that the values are somewhat higher for stars bluer than $g-i<0.6 \mathrm{mag}$. At $g-i>0.6 \mathrm{mag}$, the photometric estimates agree very well with the literature. The photometric metallicities follow the spectroscopic values from the DR9, suggesting that the over-estimates at blue colors are possibly caused by calibration problems of the DR9 for blue, metal-rich stars. M 67 shows a similar trend. Its mean photometric metallicity is $-0.16 \pm 0.11,0.07$ dex lower than the mean spectroscopic value from the DR9 and 0.16 dex lower than the literate value. For NGC 6791, the mean photometric metallicity is $0.28 \pm 0.16,0.08$ dex higher than the mean spectro- scopic value from the DR9 and 0.19 dex lower than the literature value. The small scatters of the photometric estimates of metallicities for the three OCs as well as the small systematic discrepancies with the literature values suggest that the photometric method of the current work is capable of providing metallicity estimates for MS stars accurate to $0.1-0.15$ dex.

\subsection{Effects of giant stars}

The metallicities presented in the current work are estimated using metallicity-dependent stellar loci constructed from MS stars. To investigate the possible systematics due to the contamination of giants, we apply the method to a sample of 1,996 giant stars selected from the Stripe 82 that are spectroscopically targeted by the SDSS and have a line-of-sight extinction $E(B-V) \leq 0.15 \mathrm{mag}$, $\log g \leq 3.5 \mathrm{dex}, 0.55 \leq g-i \leq 1.6 \mathrm{mag}, T_{\text {eff }} \geq 4,300 \mathrm{~K}$, and $-2.5 \leq[\mathrm{Fe} / \mathrm{H}] \leq 0.5$. The resultant photometric metallicities are then compared to the spectroscopic values.

The results are shown in Fig. 6. Compared to results of the MS sample, the $\chi_{\min }^{2}$ values of the giant sample are generally larger. There are $33.2,16.4$, and 3.9 per cent giant stars of $\chi_{\min }^{2}$ values larger than 3,5 , and 10 , respectively. The deduced photometric metallicities are systematically higher than the spectroscopic values by an average value of $0.13 \mathrm{dex}$, with a large dispersion of $0.25 \mathrm{dex}$, which is significantly higher than the value dispersion found for the MS sample. The systematics show small variations with $g-i$ color, but depend strongly on $[\mathrm{Fe} / \mathrm{H}]$. The systematic differences are close to zero for $[\mathrm{Fe} / \mathrm{H}]$ between -1.0 and -0.5 , and increase to 0.5 dex at $[\mathrm{Fe} / \mathrm{H}]=-2.0$. The results suggest that the stellar loci of giant stars are different from those of MS stars, especially for metal-poor stars. In the fourth paper of this series (Yuan et al. 2015e), we will construct the metallicity dependent stellar loci for red giants, use the results to discriminate between red giants and MS stars, and to estimate photometric metallicities of red giants based on the SDSS photometry.

\subsection{Effects of binaries}

Binaries are ubiquitous in the Galaxy (e.g., Raghavan et al. 2010), and the binary fraction varies with spectral type and possibly also with metallicity (e.g., Yuan et al. 2015d). Because the colors of MS binaries generally deviate from the metallicity-dependent stellar loci of single MS stars (Yuan et al. 2015d), estimates of photometric metallicities of MS stars could be affected by binarity. In this subsection, we explore such effects.

Following Yuan et al. (2015d), we simulate the combined colors of binary systems composed of two MS stars for all possible combinations of primary stars of $g-i$ colors between $0.3-1.6 \mathrm{mag}$ and secondary stars of $g-i$ colors between $0.3-3.0 \mathrm{mag}$, on the condition that the color of the secondary is no bluer than that of the primary. We further assume that the primary and secondary stars have the same $[\mathrm{Fe} / \mathrm{H}]$. For a binary system of given $g-i$ color set and metallicity $[\mathrm{Fe} / \mathrm{H}]$, the absolute $r$-band magnitudes, $M(r)$, of the two component stars are computed using the photometric parallax relation of Ivezić et al. [2008; Eq. (A7)]. The $u-g, g-r, r-i$, and $i-z$ colors of the two components are computed using the metallicity-dependent stellar loci determined in 

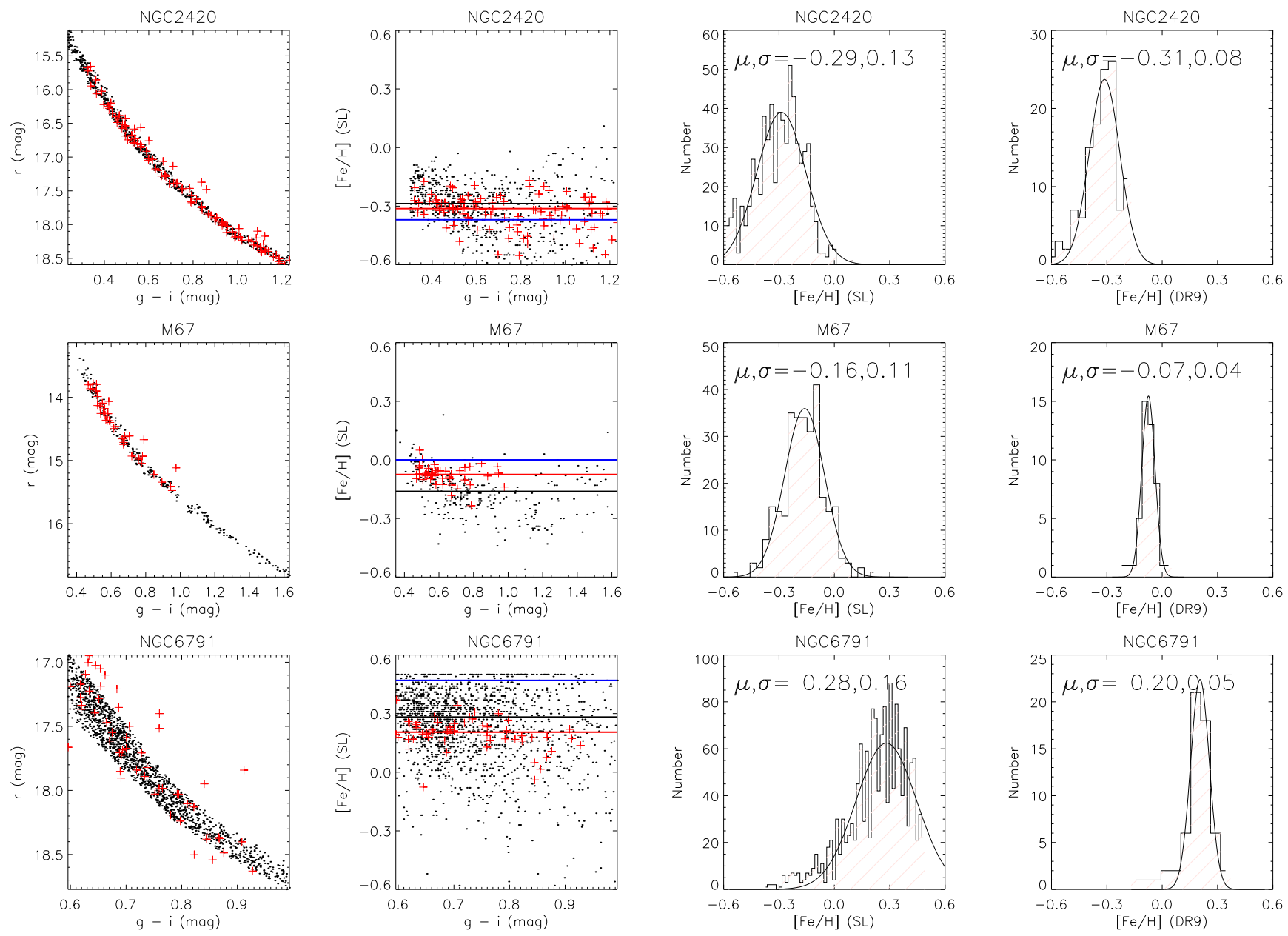

Figure 5. Comparison of photometric metallicities of MS stars of OCs NGC 2420 (upper panels), M 67 (middle panels), and NGC 6791 (lower panels) with other estimates. Column 1: $(g-i)$ versus $r$ color-magnitude diagram of the selected MS stars in the three OCs. Red pluses denote member stars spectroscopically targeted by the SDSS. Note the outliers in the $(g-i)$ versus $r$ diagram of M 67 are binaries. Column 2: Photometric metallicities plotted against color $g-i$. Red pluses denote member stars spectroscopically observed by the SDSS. The black, red, and blue lines denote the mean photometric metallicities, the mean spectroscopic metallicities from the DR9, and the literature values, respectively. Column 3: Histogram distributions of photometric metallicities. Column 4: Histogram distributions of spectroscopic metallicities from SDSS DR9. In the 3rd and 4th columns, also over-plotted are Gaussian fits to the distributions, with the central values and dispersions of the fits labeled.
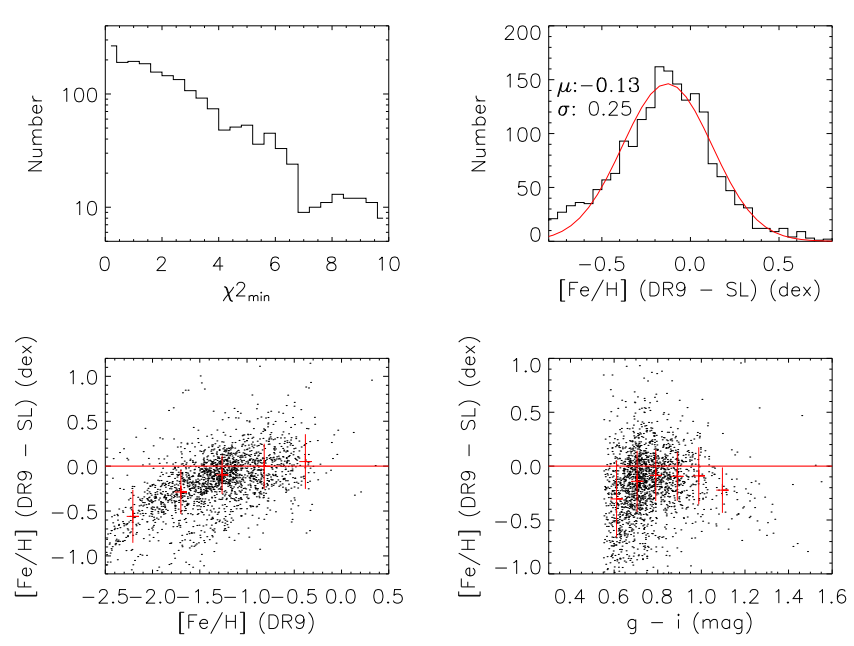

Figure 6. Same as to Fig. 2 but for the sample of giant stars.

Paper I for $g-i \leq 1.6 \mathrm{mag}$ and using the stellar loci of Covey et al. (2007) for $g-i>1.6 \mathrm{mag}$. The absolute $r$-band magnitudes and $u-g, g-r, r-i$, and $i-z$ colors are then used to derive the absolute magnitudes in $u, g, i$, and $z$ bands. Given the absolute magnitudes in $u, g, r, i$, and $z$ bands of the two components, the magnitudes and colors of the binary system are calculated. Then we use the combined colors to derive the photometric metallicity of the binary system, assuming that the errors of 0.025 , $0.015,0.015,0.02$ and $0.015 \mathrm{mag}$ in colors $u-g, g-r, r-i$, $i-z$, and $g-i$, respectively. The differences between the true and derived values of $[\mathrm{Fe} / \mathrm{H}]$ are then deduced and plotted in Fig. 7 as a function of the $g-i$ colors of the primary and secondary stars, respectively. Three sets of simulations are performed, for $[\mathrm{Fe} / \mathrm{H}]=0.0,-1.0$, and -2.0 , respectively.

Fig. 7 shows that: 1) The photometric metallicities deduced for the simulated binaries are typically more metal-poor than the true values, consistent with the fact that the combined colors are typically bluer in $u-g$ and $g-r$ and redder in $r-i$ and $i-z$ than those predicted for single stars. The systematics are in fact the underlying cause responsible for the asymmetric distributions of the differences between the photometric and spectroscopic 
metallicities of the test MS sample (c.f., top right panel of Fig. 2); 2) The systematics are however relatively small. The median differences amount to only $-0.11,-0.15$, and -0.07 dex for $[\mathrm{Fe} / \mathrm{H}]=0.0,-1.0$, and -2.0 , respectively. The maximum differences are about 0.35 dex for $[\mathrm{Fe} / \mathrm{H}]=0.0$ and $0.5 \operatorname{dex}$ for $[\mathrm{Fe} / \mathrm{H}]=-1.0$ and -2.0 . As expected, the differences are close to nil when the primary and secondary stars have close to unity or very small mass ratios. The differences reach the maximum values at certain combinations of the colors of the component stars. The results suggest that MS binaries can mimic the colors of more metal-poor (single) stars. This effect should be kept in mind when searching for very metal-poor stars from photometry alone. Note that the artifacts at $g-i \sim 1.6 \mathrm{mag}$ of the secondary are caused by the different sets of stellar loci used below and above this color. The artifacts at the top right corner of each panel are due to the fact that the combined $g-i$ colors of the simulated systems become redder than $1.6 \mathrm{mag}$, and are thus beyond the color range that can be dealt with the method presented in the current work. Due to the problems in $u-g$ color of the metallicity-dependent stellar loci at $g-i<0.4 \mathrm{mag}$, the simulated differences in $[\mathrm{Fe} / \mathrm{H}]$ are probably unrealistic at $g-i<0.4 \mathrm{mag}$.

\section{RESULTS}

We now apply the technique to the whole selected photometric sample consisting of about 0.5 million stars of Stripe 82 and estimate their photometric metallicities. With the newly derived photometric metallicities, the photometric distances of the stars are then calculated using the photometric parallax relation of Ivezić et al. [2008; Eq. (A7)]. The distance errors are also estimated, taking into account the uncertainties in $g-i$ colors, those in photometric metallicities, and the intrinsic scatter of the photometric parallax relation $(0.13 \mathrm{mag})$. The data are accessible from http://162.105.156.249/site/Stripe82/, along with a descriptive readme.txt file. About 91, 72, and 53 per cent of the sample stars are brighter than $r=20.5,19.5$, and $18.5 \mathrm{mag}$, respectively. In the calculations, we have assumed that all stars are single dwarfs. Using the Besançcon Galactic model (Robin et al. 2003), we estimate the fraction of giant stars (including sub-giants) in the sample is about 4 per cent, in agreement with the estimate of Jurić et al. (2008). The fraction is slightly higher for brighter stars. The binary fraction as a function of stellar colors and metallicities has been determined by Yuan et al. (2015d). One should keep in mind the small contamination of giant stars and the possible effects of binaries when using the sample.

The top panel of Fig. 8 plots histogram distribution of $\chi_{\min }^{2}$ for the sample. For the whole sample, 20.0, 9.2, and 2.3 per cent of the stars show a $\chi_{\min }^{2}$ value larger than 3 , 5 , and 10, respectively. The fractions are about two times larger than the corresponding values of the test sample, due to the contamination of giant stars. The fractions first decrease slightly towards brighter stars, with the corresponding percentage being 18.0, 8.0, and 2.0 per cent for stars brighter than $r=20.5$ mag and being 16.2, 7.2 , and 1.8 per cent for stars brighter than $r=19.5 \mathrm{mag}$. However, the fractions then increase, probably due to a larger fraction of giants amongst bright stars, with the corresponding percentage being $16.2,7.5$, and 2.1 per cent for stars brighter than $r=18.5 \mathrm{mag}$.

The top middle panel of Fig. 8 shows that the metallicity distribution of the sample peaks around -0.45 , and have an extended tail of metal-poor stars. Another peak around -2.25 is an artifact and caused by the systematics of the method for blue, metal-poor stars, as discussed in Section 3.1. The fraction of metal-poor stars decreases rapidly as the sample stars become brighter (closer). The trend is also seen in Fig. 9, which plots the photometric metallicities against $g-i$ colors for stars of different magnitude ranges. Fig. 9 also shows that stars of different colors exhibit different metallicity distributions, largely because stars of different colors have different intrinsic luminosities and thus probe different depths. Note that the branch of stars in the bottom left corner is also an artifact and caused by the same systematics of the method. The numbers of very metal-poor stars, defined as those of $[\mathrm{Fe} / \mathrm{H}]<-2.0$ and $\chi_{\min }^{2}<3.0$, are $210,733,2,487$, and 9,636 to a limiting magnitude of $r=16.5,17.5,18.5$, and $19.5 \mathrm{mag}$, respectively. The top right panel of Fig. 8 plots histogram distribution of the errors of photometric metallicities of the sample. The median values are 0.19 , $0.16,0.11$, and 0.085 dex for the whole sample, and for those stars that are brighter than $r=20.5,19.5$, and $18.5 \mathrm{mag}$, respectively. About 37.6, 31.9, 16.7, and 3.4 per cent of the stars have metallicity errors larger than 0.3 dex for the aforementioned four groups of stars, respectively.

The bottom two panels of Fig. 8 plot histogram distributions of photometric distances and errors for the sample. The maximum distance reached by the whole sample is about $18 \mathrm{kpc}$, and decreases to 15,10 , and $6 \mathrm{kpc}$ for stars brighter than $r=20.5,19.5$, and $18.5 \mathrm{mag}$, respectively. The median distance errors are 8.8, 8.4, 7.8, and 7.3 per cent for the aforementioned four groups of stars, respectively. For the four groups of stars, about 40.2, $35.9,25.8$, and 8.9 per cent of them have distance errors larger than 10 per cent, respectively, and only 7.0, 5.8, 2.3 , and 0.2 per cent of them have distance errors larger than 20 per cent, respectively. Note that most distance errors of the sample stars are contributed by the intrinsic scatter of the photometric parallax relation of Ivezić et al. (2008). If one neglects the intrinsic scatter of the relation, the median distance errors reduce to $6.1,5.6$, 4.6 , and 3.8 per cent for the four groups of stars, respectively. It implies that if one selects a sub-sample of stars of a narrow range in color and metallicity, then the errors of their (relative) distances can be quite small. The distributions in the $R-Z$ plane is shown in Fig. 10. The distributions are different for stars of different metallicity ranges, with the more metal-rich stars being closer to the Galactic plane.

The sample also contains a number of targets of abnormal colors, exhibiting very large values of $\chi_{\min }^{2}$. Most of them are faint white-dwarf-main-sequence binaries. Giant stars and F turnoff stars of high metallicities, in spite of their small numbers in the sample, also show very large values of $\chi_{\min }^{2}$.

\section{SUMMARY}

In this paper, we present a method to estimate photometric metallicities for MS stars, by simultaneously fitting the dereddened colors in $u-g, g-r, r-i$, and $i-z$ with those predicted by the metallicity-dependent 

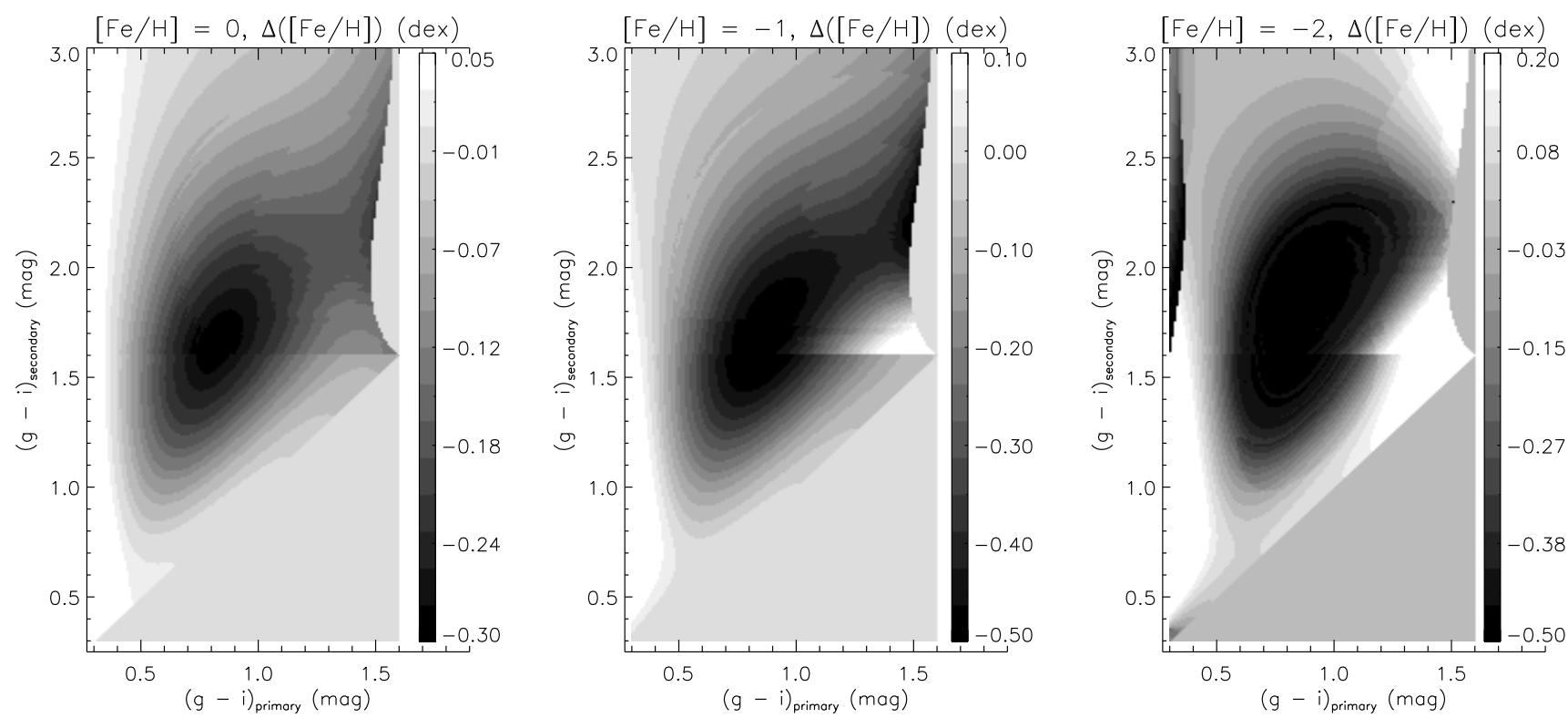

Figure 7. Distributions of the differences between the true and photometric estimates of metallicity as a function of $g-i$ colors of the primary and secondary stars of the simulated binary systems. The binaries are assumed to compose of two MS single stars of the same metallicity of $[\mathrm{Fe} / \mathrm{H}]=0.0$ (left), -1.0 (middle), and -2.0 (right), respectively. A colorbar is over-plotted by the side in each case. The stellar loci of Paper I and those of Covey et al. (2007) are used for stars of $g-i \leq 1.6$ and $>1.6$ mag, respectively. The errors in colors $u-g, g-r, r-i, i-z$, and $g-i$ are assumed to be $0.025,0.015,0.015,0.02$, and 0.015 mag, respectively.
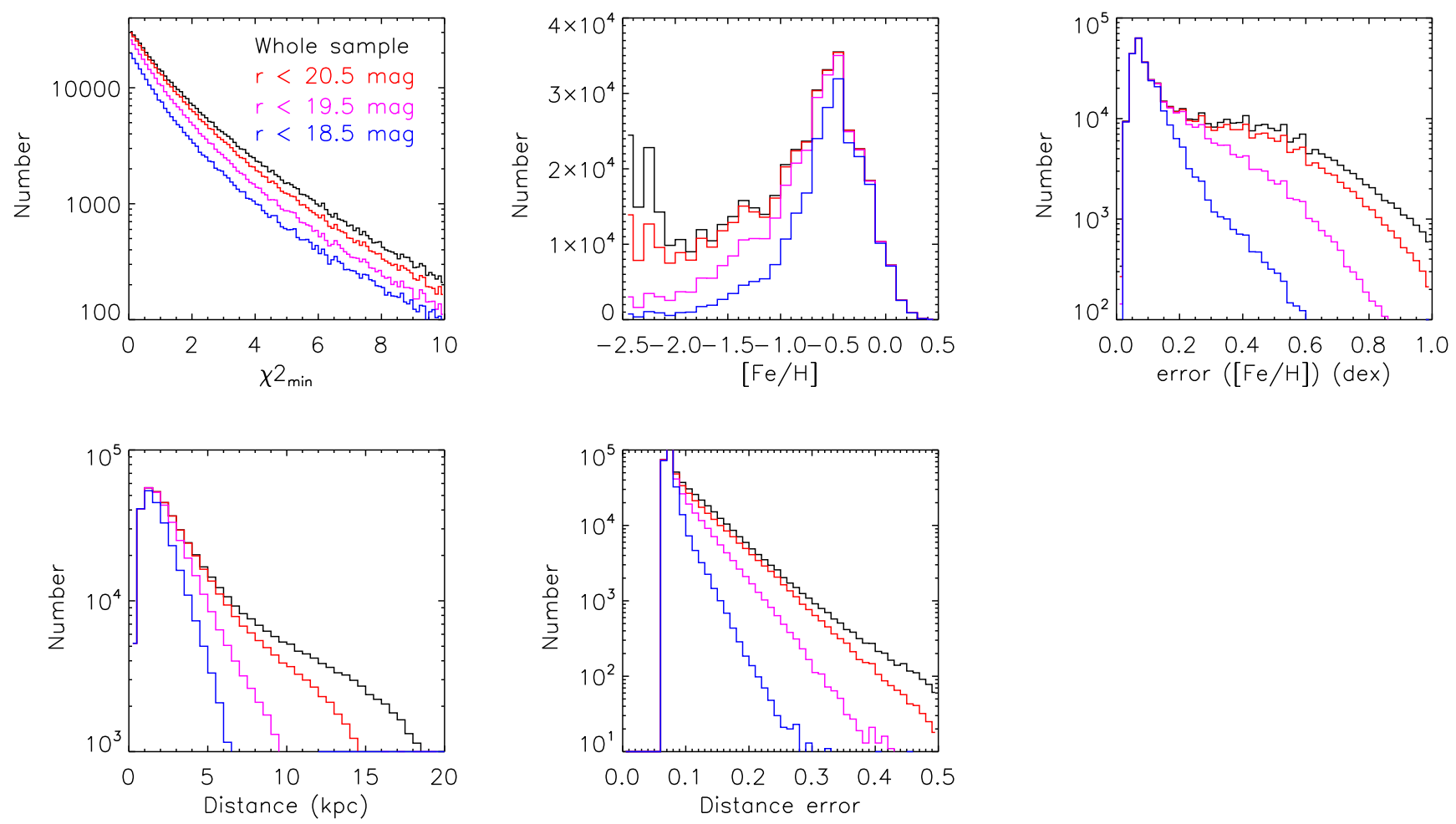

Figure 8. Histogram distributions of $\chi_{\text {min }}^{2}$ (top left), photometric metallicities (top middle) and errors (top right), photometric distances (bottom left) and errors (bottom middle) for the whole photometric sample (black), and sample stars brighter than $r=20.5$ (red), 19.5 (purple), and $18.5 \mathrm{mag}$ (blue), respectively. 


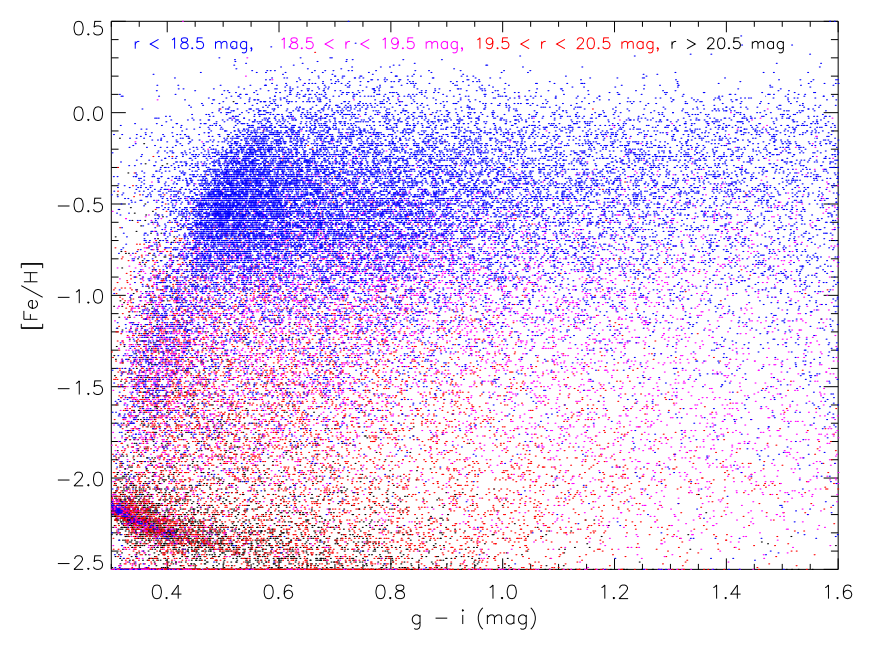

Figure 9. Distribution in the $(g-i)-[\mathrm{Fe} / \mathrm{H}]$ plane of the photometric sample. Different colors denote stars of different brightness, as marked near the top of the plot. To avoid crowdness, only onein-ten randomly selected targets are shown.

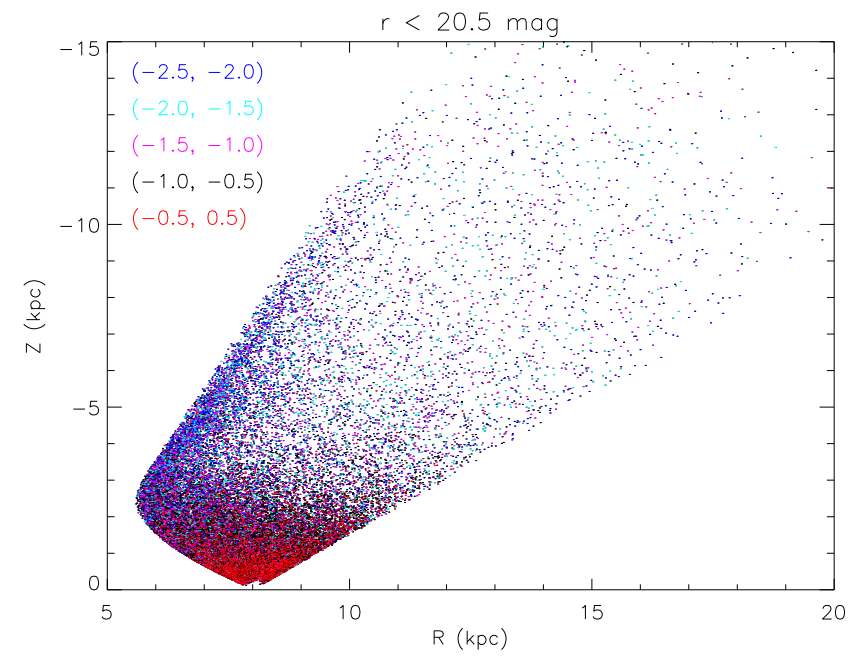

Figure 10. Spatial distribution in the $R-Z$ plane of sample of stars brighter than $r=20.5 \mathrm{mag}$. The Sun is located at $(R, Z)=$ $(8.0,0.0) \mathrm{kpc}$. Different colors denote stars of different metallicity ranges, as marked in the bottom left corner of the plot. To avoid crowdness, only one-in-ten randomly selected targets are shown.

stellar loci presented in Paper I. The method is tested using a spectroscopic sample of MS stars of Stripe 82 selected from the SDSS DR9 and three open clusters imaged and spectroscopically targeted by the SDSS. With 1 per cent photometry, the method is capable of delivering photometric metallicities precise to about $0.05,0.12$, and 0.18 dex at metallicities of $0.0,-1.0$, and -2.0 , respectively, comparable to the precision achievable with low-resolution spectroscopy for a signal-to-noise ratio of 10. Compared to the method of Ivezić et al (2008), the current method is not only more accurate, but also applicable to a wider range of color and metallicity $(0.3<$ $g-i<1.6 \mathrm{mag},-2.5<[\mathrm{Fe} / \mathrm{H}]<0.5)$, thanks to the employment of all available colors and the improved metallicity calibration of the SDSS DR9. Systematics of the method due to the possible contamination of giant stars and MS binaries are also discussed. The photometric metallicities of giant stars, when derived using the stel- lar loci of MS stars, are systematically under-estimated at $[\mathrm{Fe} / \mathrm{H}]<-1.0$. The discrepancies become larger for stars of lower metallicities. Similarly, the method underestimates the metallicities of MS binaries, by a typical amount of $0.1 \mathrm{dex}$, and up to $0.5 \mathrm{dex}$ in some extreme cases. Those systematics should be kept in mind when using the sample to search for very metal-poor stars.

We apply the method to a photometric sample of about 0.5 million stars in the Stripe 82 region that have $u, g, r, i$, and $z$ magnitudes and $g-i$ colors between $0.3-1.6$ mag. About 91, 72, and 53 per cent of the sample stars are brighter than $r=20.5,19.5$, and $18.5 \mathrm{mag}$, respectively. The median metallicity errors obtained are $0.19,0.16$, 0.11 , and 0.085 dex for the whole sample, and for stars brighter than $R=20.5,19.5$ and $18.5 \mathrm{mag}$, respectively. The metallicity distribution of the sample peaks around -0.45 , and has a tail extending to lower metallicities. The numbers of very metal-poor stars, defined as those of $[\mathrm{Fe} / \mathrm{H}]<-2.0$ and $\chi_{\min }^{2}<3.0$, count $210,733,2,487$, and 9,636 to a limiting magnitude of $r=16.5,17.5,18.5$, and $19.5 \mathrm{mag}$, respectively. With the newly deduced photometric metallicities, photometric distances are also calculated using the photometric parallax relation of Ivezić et al. (2008). The maximum distances probed by the aforementioned four group of stars are about 18, 15, 10, and $6 \mathrm{kpc}$, respectively. The median distance errors, estimated by taking into account contributions from the uncertainties of photometry metallicity, measurements of color $g-i$, and from the scatter of the photometric parallax relation, are $8.8,8.4,7.8$, and 7.3 per cent for the four groups of stars, respectively.

With accurate photometric metallicities and distances, the sample in the current work provides an excellent, magnitude-limited data-set to study, for example, 1) the metallicity gradients and distributions of the different stellar populations of the Milky Way, 2) the structure of the Galactic disk(s) as a function of metallicity and spectral type, 3) the binary fraction of field stars as a function of stellar color and metallicity (Yuan et al. 2015d), 4) the selection effects of spectroscopic surveys such as the LAMOST Galactic surveys, and 5) providing detailed constraints on Galactic models such as the Besancon model (Robin et al. 2003).

The light-motion curve catalog of Bramich et al. (2008) contains proper motions for about 1 million stars down to a limiting magnitude of $r \sim 21.5 \mathrm{mag}$, thus includes the majority sample stars of the current work. With proper motions from Bramich et al. (2008) and radial velocity measurements from LAMOST and other facilities, the current sample provides a valuable dataset to study the kinematics (e.g., Smith et al. 2009a) and sub-structures of the Galactic disk and halo (e.g., Smith et al. 2009b), and to constrain the local dark matter density (e.g., Zhang et al. 2013).

The current sample probes mainly the inner disk and halo of the Galaxy, thus is complementary to the LAMOST Spectroscopic Survey of the Galactic Anti-center (Liu et al. 2014; Yuan et al. 2015a) that targets the Galactic outer disk. The sample is accessible from http://162.105.156.249/site/Stripe82/ along with a descriptive readme.txt file.

Acknowledgments We would like to thank the referee 
for his/her useful comments. This work is supported by National Key Basic Research Program of China 2014CB845700, NSFC grant 11443006, and China Postdoctoral Science special Foundation 2014T70011. This work has made use of data products from the Sloan Digital Sky Survey.

Funding for SDSS-III has been provided by the Alfred P. Sloan Foundation, the Participating Institutions, the National Science Foundation, and the U.S. Department of Energy Office of Science. The SDSS-III web site is http://www.sdss3.org/

SDSS-III is managed by the Astrophysical Research Consortium for the Participating Institutions of the SDSS-III Collaboration including the University of Arizona, the Brazilian Participation Group, Brookhaven National Laboratory, Carnegie Mellon University, University of Florida, the French Participation Group, the German Participation Group, Harvard University, the Instituto de Astrofisica de Canarias, the Michigan State/Notre Dame/JINA Participation Group, Johns Hopkins University, Lawrence Berkeley National Laboratory, Max Planck Institute for Astrophysics, Max Planck Institute for Extraterrestrial Physics, New Mexico State University, New York University, Ohio State University, Pennsylvania State University, University of Portsmouth, Princeton University, the Spanish Participation Group, University of Tokyo, University of Utah, Vanderbilt University, University of Virginia, University of Washington, and Yale University.

\section{REFERENCES}

Ahn, C. P., et al. 2012, ApJS, 203, 21

Allende Prieto, C., Sivarani, T., Beers, T. C., et al. 2008, AJ, 136, 2070

An, D., Beers, T. C., Johnson, J. A., et al. 2013, ApJ, 763, 65

An, D., Johnson, J. A., Clem, J. L., et al. 2008, ApJS, 179, 326

An, D., Terndrup, D. M., Pinsonneault, M. H., et al. 2007, ApJ, 655,233

Anthony-Twarog, B. J., Tanner, D., Cracraft, M., \& Twarog, B. A. 2006, AJ, 131, 461

Avni Y., 1976, ApJ, 210, 642

Bramich, D. M., Vidrih, S., Wyrzykowski, L., et al. 2008, MNRAS, 386, 887

Carretta, E., Bragaglia, A., \& Gratton, R. G. 2007, A\&A, 473 , 129

Casagrande, L., Schönrich, R., Asplund, M., et al. 2011, A\&A, 530, A138

Chen, B.-Q., Liu, X.-W., Yuan, H.-B., et al. 2014, MNRAS, 443, 1192
Covey, K. R., Ivezić, Ž., Schlegel, D., et al. 2007, AJ, 134, 2398

Cui, X.-Q., Zhao, Y.-H., Chu, Y.-Q, et al. 2012, RAA, 12, 1197

Davenport, J. R. A., et al. 2014, arXiv:1403.1875

Deng, L., Newberg, H., Liu, C., et al. 2012, RAA, 12, 735

Green, G. M., et al. 2014, ApJ, 783, 114

Ivezić, Ž., Sesar, B., Jurić, M., et al. 2008, ApJ, 684, 287

Ivezić, Ž., Smith, J. A., Miknaitis, G., et al. 2007, AJ, 134, 973

Jurić, M., Ivezić, Ž., Brooks, A., et al. 2008, ApJ, 673, 864

Lee, Y. S., Beers, T. C., Sivarani, T., et al. 2008a, AJ, 136, 2022

Lee, Y. S., Beers, T. C., Sivarani, T., et al. 2008b, AJ, 136, 2050

Liu, X.-W., Yuan, H.-B, Huo, Z.-Y., et al., 2014, in Feltzing S.,

Zhao G., Walton N., Whitelock P., eds, Proc. IAU Symp. 298, Setting the scene for Gaia and LAMOST, Cambridge

University Press, pp. 310-321, preprint (arXiv: 1306.5376)

Peng, X., Du, C., Wu, Z., Ma, J., \& Zhou, X. 2013, MNRAS, 434, 3165

Raghavan, D., McAlister, H. A., Henry, T. J., et al. 2010, ApJS, 190, 1

Rebassa-Mansergas, A., et al. 2014, MNRAS, submitted

Robin, A. C., Reylé, C., Derrière, S., \& Picaud, S. 2003, A\&A, 409, 523

Sandage, A., \& Smith, L. L. 1963, ApJ, 137, 1057

Schlafly, E. F., et al. 2010, ApJ, 725, 1175

Schlegel, D. J., Finkbeiner, D. P., \& Davis, M. 1998, ApJ, 500, 525

Schlesinger, K. J., Johnson, J. A., Rockosi, C. M., et al. 2012, ApJ, 761, 160

Smith, M. C., Evans, N. W., Belokurov, V., et al. 2009, MNRAS, 399,1223

Smith, M. C., Wyn Evans, N., \& An, J. H. 2009, ApJ, 698, 1110

Smolinski, J. P., Lee, Y. S., Beers, T. C., et al. 2011, AJ, 141, 89

Steinmetz, M., Zwitter, T., Siebert, A., et al. 2006, AJ, 132, 1645

Wall J. V., 1996, QJRAS, 37, 519

Wallerstein, G. 1962, ApJS, 6, 407

Xiang, M.-S., Liu, X.-W., Yuan, H.-B., et al.,2015, MNRAS, in press

Yanny, B., et al. 2009, AJ, 137, 4377

York, D. G., et al. 2000, AJ, 120, 1579

Yuan, H. B., Liu, X.-W., Huo, Z.-Y., et al. 2015a, MNRAS, in press

Yuan, H.-B., Liu, X.-W., \& Xiang, M.-S. 2013, MNRAS, 430, 2188

Yuan, H. B., Liu, X.-W., Xiang, M.-S., Huang, Y., \& Chen, B.-Q., 2015b, ApJ, 799, 133

Yuan, H. B., Liu, X.-W., Xiang, M.-S., Huang, Y., \& Chen, B.-Q., 2015c, ApJ, 799, 134

Yuan, H. B., Liu, X.-W., Xiang, M.-S., Huang, Y., \& Chen, B.-Q., 2015d, ApJ, 799, 135

Yuan, H. B., Liu, X.-W., Xiang, M.-S., Huang, Y., \& Chen, B.-Q., 2015e, ApJ, submitted

Zhang, L., Rix, H.-W., van de Ven, G., et al. 2013, ApJ, 772, 108

Zhao, G., Zhao, Y.-H., Chu, Y.-Q., et al. 2012, Research in Astronomy and Astrophysics, 12, 723 